\title{
Ranging algebraically with more observations than unknowns
}

\author{
Joseph L. Awange ${ }^{1}$, Yoichi Fukuda ${ }^{1}$, Shuzo Takemoto ${ }^{1}$, Ismail L. Ateya ${ }^{1}$, and Erik W. Grafarend ${ }^{2}$ \\ ${ }^{1}$ Department of Geophysics, Kyoto University, Kitashirakawa Oiwake-cho, Sakyo-ku, Kyoto 606-8502, Japan \\ ${ }^{2}$ Department of Geodesy and GeoInformatics, Geschwister-Scholl str. 24D, 70174 Stuttgart, Germany
}

(Received May 6, 2003; Revised July 16, 2003; Accepted July 16, 2003)

\begin{abstract}
In the recently developed Spatial Reference System that is designed to check and control the accuracy of the three-dimensional coordinate measuring machines and tooling equipment (Metronom US., Inc., Ann Arbor: http://www. metronomus.com), the coordinates of the edges of the instrument are computed from distances of the bars. The use of distances in industrial application is fast gaining momentum just as in Geodesy and in Geophysical applications and thus necessitating efficient algorithms to solve the nonlinear distance equations. Whereas the ranging problem with minimum known stations was considered in our previous contribution in the same Journal, the present contribution extends to the case where one is faced with many distance observations than unknowns (overdetermined case) as is usually the case in practise. Using the Gauss-Jacobi Combinatorial approach, we demonstrate how one can proceed to position without reverting to iterative and linearizing procedures such as Newton's or Least Squares approach.
\end{abstract}

Key words: Overdetermined planar ranging, overdetermined three-dimensional ranging, Gauss-Jacobi combinatorial algorithm, Groebner basis, Multipolynomial resultant, Least Squares.

\section{Introduction}

In Awange et al. (2003), we highlighted the importance of ranging problem and presented algebraic procedures of reduced Groebner basis and Multipolynomial resultant approaches for solving the minimum ranging problem directly or explicitly in a closed form. In practise however, one is often faced with a situation where more observations than unknowns exist. In such cases, the usual practise is often to rely on linearization or iterative procedures.

The bottleneck to the procedures of linearization and iterative of relying heavily on the initial starting values to obtain global minimum and faster convergence has been treated in recent works of Xu $(2002,2003)$. In general, the problem of nonlinear adjustment has been considered by among others Teunissen (1990), Grafarend and Schaffrin $(1989,1991)$ who extend the work of Krarup (1982) on nonlinear adjustment with respect to geocentric interpretation and Guolin (2000) who presents a procedure that uses F-distributions to test whether the nonlinear model can be linearized or not. An extensive analysis of the nonlinear problem with an elaborate literature review has been presented by Lohse (1994) and Mautz (2001).

The common features with the non-algebraic approaches in solving nonlinear problems is that they all have to do with some starting values, linearization of the observation equations and iterations. Although the issue of approximate starting values has been addressed in the works of Xu (2002, 2003), the algebraic (approach e.g. Awange, submitted) enjoys the advantage that all the requirements listed above for non-algebraic approaches are immaterial. The

Copy right(C) The Society of Geomagnetism and Earth, Planetary and Space Sciences (SGEPSS); The Seismological Society of Japan; The Volcanological Society of Japan; The Geodetic Society of Japan; The Japanese Society for Planetary Sciences. nonlinear problem is solved in an exact form with linearization permitted only during the formation of the variancecovariance matrix to generate the weight matrix of the pseudo-observations. No starting values, linearization of the observation equations, iterations and convergence conditions are required. The only requirement is to be able to solve in a closed (exact) form systems of nonlinear equations, a condition already presented in Awange et al. (2003). Other added advantages of the algebraic approach is that during the solution of the combinatorial subsets, any presence of outlying observations can be diagnosed (e.g. Awange, in press) and also that it provides an independent approach that can be used to control the non-algebraic procedures.

The present contribution extends on the work of Awange et al. (2003) by employing the Gauss-Jacobi Combinatorial approach presented in Awange and Grafarend (2003, in press) to solve without linearization or iteration the overdetermined ranging problem. We employ the algebraic approaches presented in Awange et al. (2003) as computing engine. The contribution provides efficient techniques based on algebra that have already been applied in Geodesy as evidenced in the work of Awange (2002) and Awange and Grafarend (2002a, 2002b, 2003). The technique could also be applied in Geophysics and also in Industrial applications e.g. in the work of Jurisch et al. (2003).

We organize the present contribution as follows; in Section 2, we present a summary of the Gauss-Combinatorial algorithm while in Section 3, the overdetermined twodimensional ranging problem is solved. Section 4 considers the three-dimensional case, while Section 5 concludes the study. 


\section{Gauss-Jacobi Algorithm}

The Gauss-Jacobi combinatorial algorithm named after C. F. Gauss (Awange and Grafarend 2003, appendix A) and C. G. I. Jacobi operates in three phases. In the first phase, one forms minimal combinations of the nonlinear equations from the observation sample that are solved in a closed form using the Groebner basis or Multipolynomial resultant algebraic techniques discussed in Awange et al. (2003) to obtain the desired combinatorial solutions. The net result is that one ends with pseudo-observations, which are within the solution space of the desired values. This first phase in essence projects a nonlinear case into a linear case. The process of solving the minimal combinatorial subsets is akin to the Gauss-elimination technique used for solving linear system of equations.

Once the first phase is successfully carried out with the solutions of the various subsets acting as pseudo-observations, the nonlinear variance-covariancelerror propagation has to be carried out in the second phase to obtain the weight matrix of the pseudo-observations. This then requires that the stochasticity of the initial observational sample be known in order to propagate them to the pseudo-observations.

The final phase entails the adjustment step, which is performed to obtain the barycentric values. Since the pseudoobservations are linearly independent, the special linear Gauss-Markov model (Awange and Grafarend, 2003, definition 2-1) is employed.

Stepwise, the Gauss-Jacobi Combinatorial algorithm discussed in detail in Awange and Grafarend (2003) operates as following:

Step 1: Given an overdetermined system with $n$ observations in $m$ unknowns, from the $n$ observations form the

$$
k(\text { no. of combinations })=\frac{n !}{m !(n-m) !}
$$

minimal combination that comprise $m$ equations that are to be solved in closed form using the Groebner basis or Multipolynomial resultant algebraic techniques.

Step 2: Solve each set of $m$ equations from Step 1 above using either Groebner basis or Multipolynomial resultant algebraic techniques already presented in Awange et al. (2003).

Step 3: Perform the nonlinear error/variance-covariance propagation to obtain the variance-covariance matrix of the pseudo-observations obtained in the $i$-th combinatorial solutions of Step 2. Once this has been done for all the $k$ combinatorials, a unified variance-covariance/dispersion matrix for the entire pseudo-observations is computed e.g. as in equation (3-2).

Step 4: Using the pseudo-observations of Step 2 and the variance-covariance matrix from Step 3, adjust the pseudoobservations via the special linear Gauss-Markov model.

\section{Example 2-1 :}

The following example based on a linear case illustrates the principles behind the algorithm.

Consider a case where three linear equations have been given for the purpose of solving the two unknowns $(x, y)$. Three possible combinations each containing two equations necessary for solving the two unknowns in a closed form can be formed. Each of the system
Table 1. Distance observations to the unknown station $N$.

\begin{tabular}{cccc}
\hline $\begin{array}{c}\text { Pt. } \\
\text { No. }\end{array}$ & $\begin{array}{c}\text { Easting } \\
x[\mathrm{~m}]\end{array}$ & $\begin{array}{c}\text { Northing } \\
y[\mathrm{~m}]\end{array}$ & $\begin{array}{c}s_{i} \\
{[\mathrm{~m}]}\end{array}$ \\
\hline 1 & 48177.62 & 6531.28 & 611.023 \\
2 & 49600.15 & 7185.19 & 1529.482 \\
3 & 49830.93 & 5670.69 & 1323.884 \\
4 & 47863.91 & 5077.24 & 1206.524 \\
\hline
\end{tabular}

of two linear equations is either solved by substitution, graphically or matrix form to give three pairs of solutions $\left\{x_{1,2}, y_{1,2}, x_{2,3}, y_{2,3}, x_{1,3}, y_{1,3}\right\}$. The final step now involves the adjustment of these pseudo-observations $\left\{x_{1,2}, y_{1,2}, x_{2,3}, y_{2,3}, x_{1,3}, y_{1,3}\right\}$ with the weight matrix $\boldsymbol{\Sigma}$ obtained via nonlinear error/variance-covariance propagation.

Extensive exposition of the Gauss-Jacobi combinatorial algorithm is presented in Awange and Grafarend (2003, in press).

\section{Overdetermined Ranging}

\subsection{Overdetermined two-dimensional ranging}

In order to solve the overdetermined $2 \mathrm{~d}$ ranging problem, we refer to the Gauss-Jacobi combinatorial algorithm discussed in Section 2. Combinatorials are formed using (2-1) and solved in a closed form using

$$
\begin{aligned}
& e_{2} Y_{0}^{2}+e_{1} Y_{0}+e_{0}=0 \\
& f_{2} X_{0}^{2}+f_{1} X_{0}+f_{0}=0
\end{aligned}
$$

with the coefficients as given in Awange et al. (2003). For each minimal combinatorial set, one also computes in the second step the dispersion matrix of the resulting pseudoobservations using Eqs. (3-8), (3-9) and (3-10) of Box (3-1) and

$$
D\{\boldsymbol{x}\}=\boldsymbol{J}_{x}^{-1} \boldsymbol{J}_{y} \boldsymbol{\Sigma}_{y} \boldsymbol{J}_{y}^{\prime}\left(\boldsymbol{J}_{x}^{-1}\right)^{\prime}
$$

with $\boldsymbol{J}_{x}, \boldsymbol{J}_{y}$ being the partial derivatives of (3-9) and (310) with respect to $\boldsymbol{x}, \boldsymbol{y}$ respectively at the Taylor points $\left(\boldsymbol{\mu}_{x}, \boldsymbol{\mu}_{y}\right)$. The approximate values of unknown parameters $\left\{x_{1}, \ldots, x_{m}\right\} \in \boldsymbol{x}$ appearing in the Jacobi matrices $\boldsymbol{J}_{x}, \boldsymbol{J}_{y}$ are obtained from Groebner basis or Multipolynomial resultants solution of the nonlinear system of equations (3-5). Finally the pseudo-observation are adjusted by the use of special linear Gauss-Markov model in Step 3 with the unknowns estimated via Best Linear Uniformly Unbiased Estimator BLUUE (Awange and Grafarend, 2003, equation 15) as

$$
\hat{\xi}=\left(\boldsymbol{A}^{\prime} \boldsymbol{\Sigma}^{-1} \boldsymbol{A}\right)^{-1} \boldsymbol{A}^{\prime} \boldsymbol{\Sigma}^{-1} \boldsymbol{y}
$$

and the regular dispersion matrix of the estimated parameters given by

$$
D\{\hat{\xi}\}=\left(\boldsymbol{A}^{\prime} \boldsymbol{\Sigma}^{-1} \boldsymbol{A}\right)^{-1}
$$

The procedure becomes clear once we consider as example from Kahmen and Faig (1988) next. 
Box 3-1 (error propagation for planar ranging problem):

For the unknown point $P(X, Y) \in \mathbb{E}^{2}$ of the planar ranging problem, let distances $S_{1}$ and $S_{2}$ be measured to two known points $P_{1}\left(X_{1}, Y_{1}\right) \in \mathbb{E}^{2}$ and $P_{2}\left(X_{2}, Y_{2}\right) \in \mathbb{E}^{2}$ respectively, the distance equations expressed as

$$
\left[\begin{array}{l}
S_{1}^{2}=\left(X_{1}-X\right)^{2}+\left(Y_{1}-Y\right)^{2} \\
S_{2}^{2}=\left(X_{2}-X\right)^{2}+\left(Y_{2}-Y\right)^{2}
\end{array}\right.
$$

which we express in algebraic form as

$$
\left[\begin{array}{l}
f_{1}:=\left(X_{1}-X\right)^{2}+\left(Y_{1}-Y\right)^{2}-S_{1}^{2}=0 \\
f_{2}:=\left(X_{2}-X\right)^{2}+\left(Y_{2}-Y\right)^{2}-S_{2}^{2}=0
\end{array}\right.
$$

On taking total differential of (3-6) we have

$$
\left[\begin{array}{c}
d f_{1}:=2\left(X_{1}-X\right) d X_{1}-2\left(X_{1}-X\right) d X+2\left(Y_{1}-Y\right) d Y_{1}- \\
-2\left(Y_{1}-Y\right) d Y-2 S_{1} d S_{1}=0 \\
d f_{2}:=2\left(X_{2}-X\right) d X_{2}-2\left(X_{2}-X\right) d X+2\left(Y_{2}-Y\right) d Y_{2}- \\
-2\left(Y_{2}-Y\right) d Y-2 S_{2} d S_{2}=0
\end{array}\right.
$$

which on arranging the differential vector of the unknown terms $\{X, Y\}=\left\{x_{1}, x_{2}\right\} \in \boldsymbol{x}$ on the left hand side and that of the known terms $\left\{X_{1}, Y_{1}, X_{2}, Y_{2}, S_{1}, S_{2}\right\}=\left\{y_{1}, y_{2}, y_{3}, y_{4}, y_{5}, y_{6}\right\} \in \boldsymbol{y}$ on the right hand side leads to

$$
\begin{aligned}
& \boldsymbol{J}_{x}\left[\begin{array}{c}
d X \\
d Y
\end{array}\right]=\boldsymbol{J}_{y}\left[\begin{array}{l}
d S_{1} \\
d X_{1} \\
d Y_{1} \\
d S_{2} \\
d X_{2} \\
d Y_{2}
\end{array}\right] \\
& \text { with } \\
& \boldsymbol{J}_{x}=\left[\begin{array}{ll}
\frac{\partial f_{1}}{\partial X} & \frac{\partial f_{1}}{\partial Y} \\
\frac{\partial f_{2}}{\partial X} & \frac{\partial f_{2}}{\partial Y}
\end{array}\right]=\left[\begin{array}{l}
-2\left(X_{1}-X\right)-2\left(Y_{1}-Y\right) \\
-2\left(X_{2}-X\right)-2\left(Y_{2}-Y\right)
\end{array}\right] \\
& \text { and } \\
& \boldsymbol{J}_{y}=\left[\begin{array}{cccccc}
\frac{\partial f_{1}}{\partial S_{1}} & \frac{\partial f_{1}}{\partial X_{1}} & \frac{\partial f_{1}}{\partial Y_{1}} & 0 & 0 & 0 \\
0 & 0 & \frac{\partial f_{2}}{\partial S_{2}} & \frac{\partial f_{2}}{\partial X_{2}} & \frac{\partial f_{2}}{\partial Y_{2}}
\end{array}\right]= \\
& \left.=\left[\begin{array}{ccccc}
2 S_{1}-2\left(X_{1}-X\right) & -2\left(Y_{1}-Y\right) & 0 & 0 & 0 \\
0 & 0 & 0 & 2 S_{2}-2\left(X_{2}-X\right) & -2\left(Y_{2}-Y\right)
\end{array}\right]\right] \\
& D\{\boldsymbol{x}\}=\boldsymbol{\Sigma}_{x}=\left[\begin{array}{cc}
\sigma_{X}^{2} & \sigma_{X Y} \\
\sigma_{Y X} & \sigma_{Y}^{2}
\end{array}\right] \text { and } D\{\boldsymbol{y}\}=\boldsymbol{\Sigma}_{y}=\left[\begin{array}{cccccc}
\sigma_{S_{1}}^{2} & \sigma_{S_{1} X_{1}} & \sigma_{S_{1} Y_{1}} & \sigma_{S_{1} X_{2}} & \sigma_{S_{1} S_{2}} & \sigma_{S_{1} Y_{2}} \\
\sigma_{X_{1} S_{1}} & \sigma_{X_{1}}^{2} & \sigma_{X_{1} Y_{1}} & \sigma_{X_{1} S_{2}} & \sigma_{X_{1} X_{2}} & \sigma_{X_{1} Y_{2}} \\
\sigma_{Y_{1} S_{1}} & \sigma_{Y_{1} X_{1}} & \sigma_{Y_{1}}^{2} & \sigma_{Y_{1} S_{2}} & \sigma_{Y_{1} X_{2}} & \sigma_{Y_{1} Y_{2}} \\
\sigma_{S_{2} S_{1}} & \sigma_{S_{2} X_{1}} & \sigma_{S_{2} Y_{1}} & \sigma_{S_{2}}^{2} & \sigma_{S_{2} X_{2}} & \sigma_{S_{2} Y_{2}} \\
\sigma_{X_{2} S_{1}} & \sigma_{X_{2} X_{1}} & \sigma_{X_{2} Y_{1}} & \sigma_{X_{2} S_{2}} & \sigma_{X_{2}}^{2} & \sigma_{X_{2} Y_{2}} \\
\sigma_{Y_{2} S_{1}} & \sigma_{Y_{2} X_{1}} & \sigma_{Y_{2} Y_{1}} & \sigma_{Y_{2} S_{2}} & \sigma_{Y_{2} X_{2}} & \sigma_{Y_{2}}^{2}
\end{array}\right]
\end{aligned}
$$

we obtain with (3-8), (3-9) and (3-10) the dispersion $D\{\boldsymbol{x}\}=\boldsymbol{J}_{x}^{-1} \boldsymbol{J}_{y} \boldsymbol{\Sigma}_{y} \boldsymbol{J}_{y}^{\prime}\left(\boldsymbol{J}_{x}^{-1}\right)^{\prime}$ of the unknown variables $\{X, Y\}=$ $\left\{x_{1}, x_{2}\right\} \in \boldsymbol{x}$.

\section{Example:}

Let us consider the example of Kahmen and Faig (1988, pp. 240-241) where the coordinates of point $N$ are to be determined from distance observations to four points $P_{1}, P_{2}, P_{3}$ and $P_{4}$ (i.e. figure 6.4 .4 of Kahmen and Faig, ibid, p. 229). In preparation for adjustment, the distances are corrected and reduced geometrically to Gauss-Krueger projection and are as given in Table 1.

In order to test the Gauss-Jacobi combinatorial algorithm, we compute the coordinates of station $N$ and compare it 
Table 2. Position of station $N$ computed for various combinatorials.

\begin{tabular}{cccc}
\hline $\begin{array}{c}\text { Combinatorial } \\
\text { No. }\end{array}$ & $\begin{array}{c}\text { Combinatorial } \\
\text { points }\end{array}$ & $\begin{array}{c}x \\
{[\mathrm{~m}]}\end{array}$ & $\begin{array}{c}y \\
{[\mathrm{~m}]}\end{array}$ \\
\hline 1 & $1-2$ & 48565.2783 & 6058.9770 \\
2 & $1-3$ & 48565.2636 & 6058.9649 \\
3 & $1-4$ & 48565.2701 & 6058.9702 \\
4 & $2-3$ & 48565.2697 & 6058.9849 \\
5 & $2-4$ & 48565.3402 & 6058.9201 \\
6 & $2-5$ & 48565.2661 & 6058.9731 \\
\hline
\end{tabular}

Table 3. Position of station $N$ after adjustments.

\begin{tabular}{lcccccc}
\hline \multicolumn{1}{c}{ Approach } & $x(m)$ & $y(m)$ & $\sigma_{x}(m)$ & $\sigma_{y}(m)$ & $\Delta_{x}(m)$ & $\Delta_{y}(m)$ \\
\hline Least Squares & 48565.2700 & 6058.9750 & 0.006 & 0.006 & - & - \\
Gauss-Jacobi (BLUUE) & 48565.2709 & 6058.9750 & 0.0032 & 0.0034 & -0.0009 & 0.0000 \\
Gauss-Jacobi (arithmetic mean) & 48565.2813 & 6058.9650 & - & - & -0.01133 & 0.0100 \\
\hline
\end{tabular}

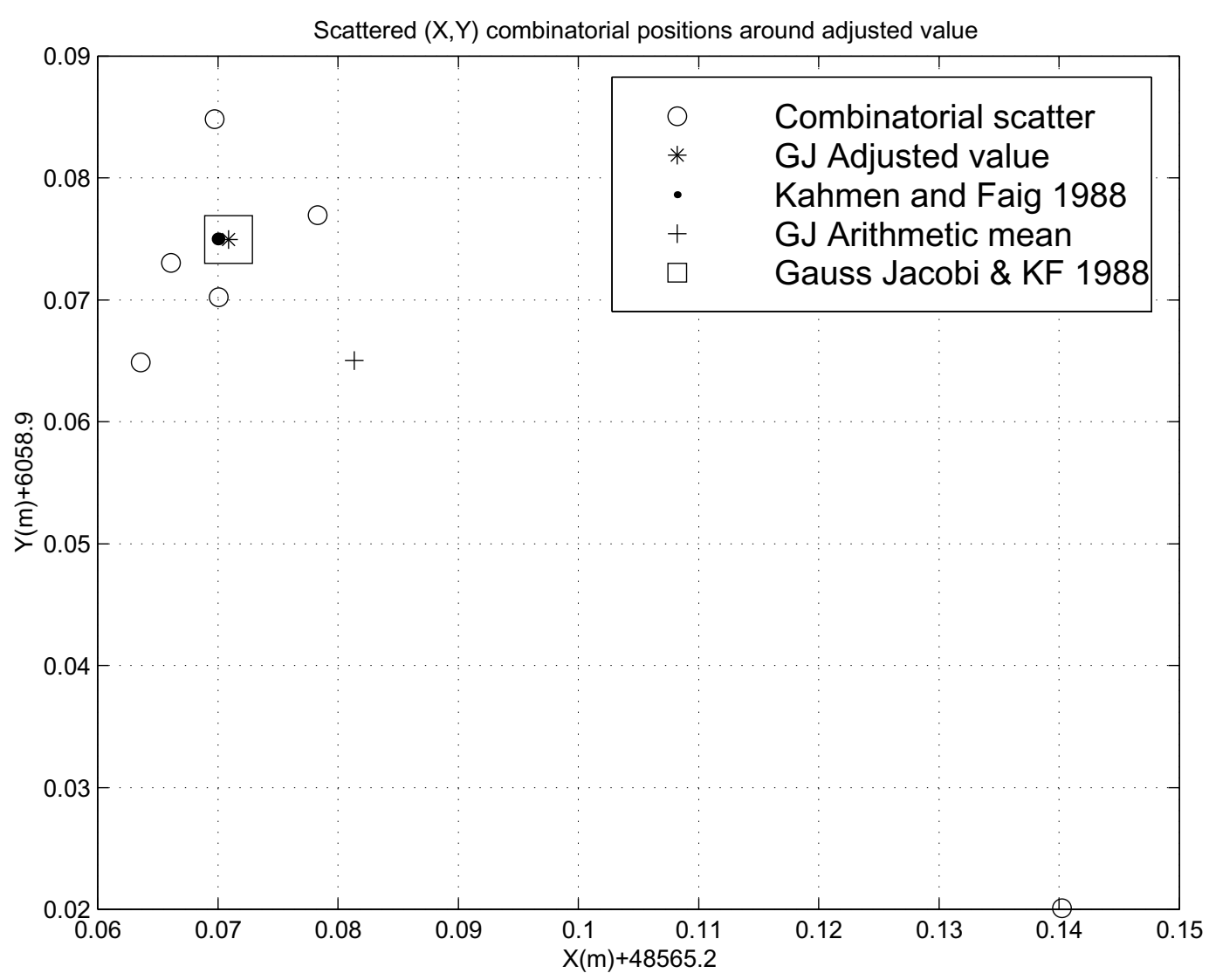

Fig. 1. Plot of the position of $N$ from various approaches.

with the Least Squares value of Kahmen and Faig (1988, p. 242). From (2-1), six combinatorials in the minimal sense are formed with each combinatorial solved for $\{x, y\}_{N}$ for point $N$ using (3-1) as discussed in Awange et al. (2003). The combinatorial solutions are presented in Table 2.

The barycentric coordinate of station $N$ is now obtained either by (a) simply taking the arithmetic mean of the combinatorial solutions in columns 3 and 4 of Table 2 (an approach which does not take into account full information in terms of the variance-covariance matrix) of the pseudoobservations or (b) by using special linear Gauss-Markov model through the estimation by the Best Linear Uniformly Unbiased Estimator BLUUE in Eq. (3-3) and the dispersion obtained by (3-4). The results are presented in Table 3 and plotted in Fig. 1. In Table 3, we present the coordinates $\{x, y\}$ of station $N$ obtained using the Least Squares approach in Kahmen and Faig (1988), Gauss-Jacobi combinatorial (BLUUE) and the Gauss-Jacobi combinatorial (arith- 


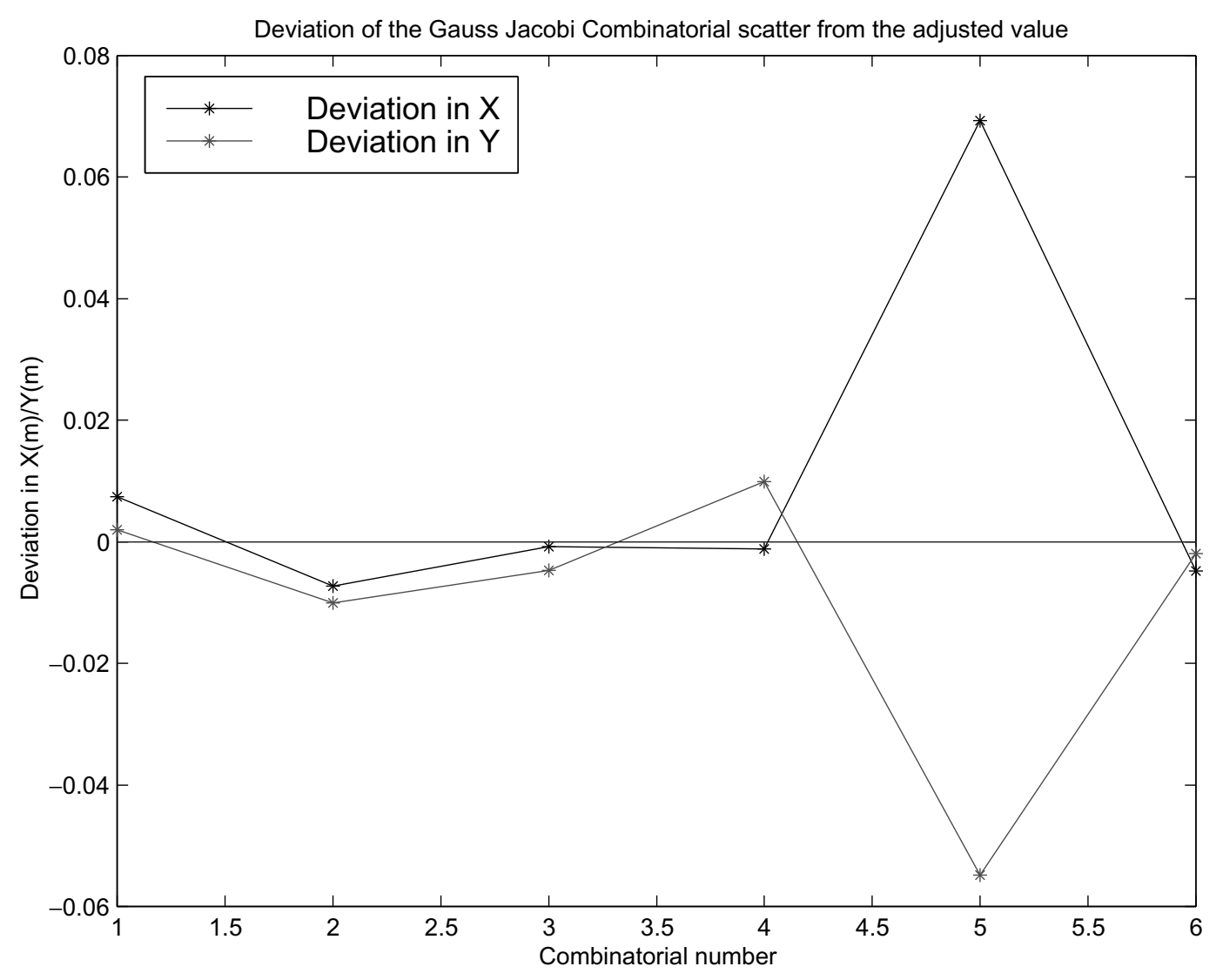

Fig. 2. Deviations of the combinatorial scatter from the BLUUE adjusted position of $N$.

metic mean) in columns 2 and 3 with their respective standard deviations $\left\{\sigma_{x}, \sigma_{y}\right\}$ in columns 4 and 5. In columns 6 and 7 , the deviations $\left\{\Delta_{x}, \Delta_{x}\right\}$ of the computed coordinates of station $N$ using the Gauss-Jacobi combinatorial (BLUUE and arithmetic mean) from the values of Kahmen and Faig (1988) are presented. The deviations of the exact solutions of each combination (columns 3 and 4 of Table 2) from the adjusted values of Best Linear Uniformly Unbiased Estimator BLUUE (i.e. second raw of Table 3) obtained using Eq. (3-3) are plotted in Fig. 2.

From the results in Table 3 and Fig. 1, it is seen that when the full information of the observation is taken into account via the nonlinear error/variance-covariance propagation and the parameters estimated via BLUUE for the linear Gauss-Markov model in the final step for the barycentric coordinates, the Gauss-Jacobi combinatorial algorithm gives the same results as Least Squares adjustment (from Kahmen and Faig, 1988). In addition to giving the barycentric coordinates, the Gauss-Jacobi algorithm can accurately pin point a poor combinatorial geometry (e.g. combination 5) although this is taken care of through weighting. Figure 1 shows the combinatorial scatter denoted by $\{0\}$ and the Gauss-Jacobi combinatorial adjusted value with $\{*\}$. Least Squares estimation from Kahmen and Faig (1988) by $\{\bullet\}$ and the arithmetic mean by $\{+\}$. One notes that the estimates from Gauss-Jacobi's BLUUE $\{*\}$ and Least Squares estimation from Kahmen and Faig (1988) almost coincide. In the Figure, both estimates are encircled by $\{\square\}$ for clarity purposes.
These results indicate the capability of the Gauss-Jacobi combinatorial algorithm to solve overdetermined planar ranging problems.

\subsection{Overdetermined three-dimensional ranging}

Having solved the overdetermined planar ranging problem in Section 3.1, we extend the use of the Gauss-Jacobi combinatorial algorithm to solve the overdetermined threedimensional ranging problem in this section. An example based on the test network "Stuttgart Central" in Fig. 3 is considered.

\section{Example}

The test network "Stuttgart Central" in Fig. 3 consists of distance observations from station $K_{1}$ to seven other stations. Desired are the three-dimensional coordinates $X, Y, Z$ of the unknown point $K_{1}$ obtained by solving three nonlinear distance observation equations in closed form discussed in Awange et al. (2003).

From Fig. 3 and using (2-1), 35 combinatorial subsets are formed whose systems of nonlinear distance equations are solved for the position $X, Y, Z$ of the unknown point $K_{1}$ in closed form using either Groebner basis approach derived equations (e.g. Awange et al., 2003, box 3-4)

$$
Y=\frac{\left\{\left(a_{12} c_{02}-a_{02} c_{12}\right) Z+a_{12} f_{02}-a_{02} f_{12}\right\}}{\left(a_{02} b_{12}-a_{12} b_{02}\right)}
$$

and

$$
X=\frac{-\left(b_{12} Y+c_{12} Z+f_{12}\right)}{a_{12}}
$$




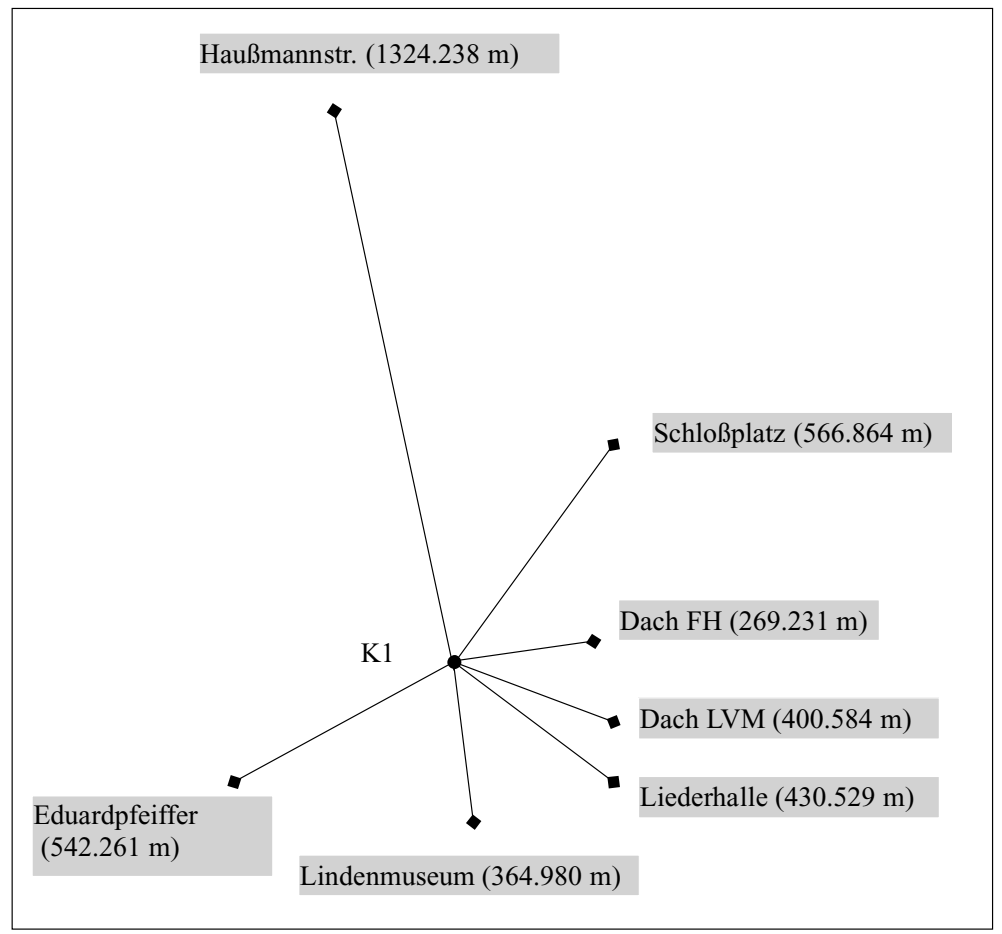

Fig. 3. Test network "Stuttgart Central”.

or

$$
X=\frac{\left\{\left(b_{02} c_{12}-b_{12} c_{02}\right) Z+b_{02} f_{12}-b_{12} f_{02}\right\}}{\left(a_{02} b_{12}-a_{12} b_{02}\right)}
$$

or Multipolynomial resultant approach derived equations (e.g. Awange et al., 2003, box 3-5)

$$
X=\frac{\left\{\left(b_{12} c_{02}-b_{02} c_{12}\right) Z+b_{12} f_{02}-b_{02} f_{12}\right\}}{\left(b_{02} a_{12}-b_{12} a_{02}\right)}
$$

and

$$
Y=\frac{\left\{\left(a_{12} c_{02}-a_{02} c_{12}\right) Z+a_{12} f_{02}-a_{02} f_{12}\right\}}{\left(a_{02} b_{12}-a_{12} b_{02}\right)} .
$$

35 different positions $X, Y,\left.Z\right|_{P}$ of the same point $P$ totalling to $105(35 \times 3)$ values of $X, Y, Z$ which are treated as pseudo-observations are obtained. One then proceeds in two steps as follows:

Step 1: From the 35 combinatorials obtained using (2-1), solve for $X, Y, Z$ in close form using either (3-12) and (312) or (3-14) and (3-15).

Step 2: (Error propagation to determine the dispersion matrix $\boldsymbol{\Sigma}$ based on linearized approximation):

The variance-covariance matrix is computed for each of the combinatorial set $j=1, \ldots, 35$ using error propagation. The closed form observational equations for the first combinatorial subset $j=1$ (i.e. tetrahedron $P P_{1} P_{2} P_{3}$ ) Awange et al. (2003) are written algebraically as

$$
\left\{\begin{array}{l}
f_{1}:=\left(X_{1}-X\right)^{2}+\left(Y_{1}-Y\right)^{2}+\left(Z_{1}-Z\right)^{2}-S_{1}^{2} \\
f_{2}:=\left(X_{2}-X\right)^{2}+\left(Y_{2}-Y\right)^{2}+\left(Z_{2}-Z\right)^{2}-S_{2}^{2} \\
f_{3}:=\left(X_{3}-X\right)^{2}+\left(Y_{3}-Y\right)^{2}+\left(Z_{3}-Z\right)^{2}-S_{3}^{2}
\end{array}\right.
$$

where $S_{i}^{j}|i \in\{1,2,3\}| j=1$ are the distances between known GPS stations $P_{i} \in \mathbb{E}^{3} \mid i \in\{1,2,3\}$ of the test network "Stuttgart Central" and the unknown GPS point $P \in$ $\mathbb{E}^{3}$ for first combination set $j=1$. With (3-9) and (3-10) we have the Jacobi matrices respectively as

$\boldsymbol{J}_{x}=\left[\begin{array}{lll}\frac{\partial f_{1}}{\partial X} & \frac{\partial f_{1}}{\partial Y} & \frac{\partial f_{1}}{\partial Z} \\ \frac{\partial f_{2}}{\partial X} & \frac{\partial f_{2}}{\partial Y} & \frac{\partial f_{2}}{\partial Z} \\ \frac{\partial f_{3}}{\partial X} & \frac{\partial f_{3}}{\partial Y} & \frac{\partial f_{3}}{\partial Z}\end{array}\right]=\left[\begin{array}{l}-\left(X_{1}-X\right)-\left(Y_{1}-Y\right)-\left(Z_{1}-Z\right) \\ -\left(X_{2}-X\right)-\left(Y_{2}-Y\right)-\left(Z_{2}-Z\right) \\ -\left(X_{3}-X\right)-\left(Y_{3}-Y\right)-\left(Z_{3}-Z\right)\end{array}\right]$

and

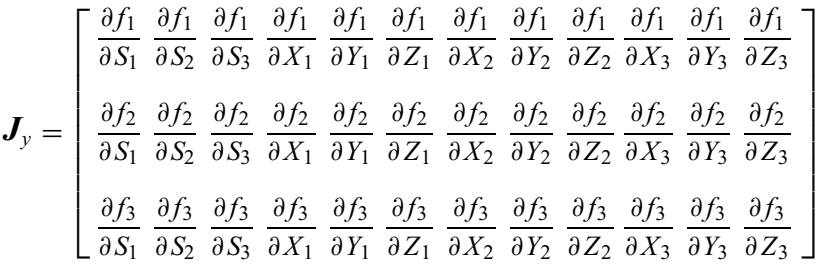

$$
\begin{aligned}
& =\left[\begin{array}{cccccccccccc}
S_{1} & 0 & 0 & j_{14} & j_{15} & j_{16} & 0 & 0 & 0 & 0 & 0 & 0 \\
0 & S_{2} & 0 & 0 & 0 & 0 & j_{27} & j_{28} & j_{29} & 0 & 0 & 0 \\
0 & 0 & S_{3} & 0 & 0 & 0 & 0 & 0 & 0 & j_{10} & j_{11} & j_{12}
\end{array}\right]
\end{aligned}
$$

where

$$
\left[\begin{array}{ccc}
j_{14}=-\left(X-X_{1}\right), & j_{15}=-\left(Y_{1}-Y\right), & j_{16}=-\left(Z_{1}-Z\right) \\
j_{27}=-\left(X_{2}-X\right), & j_{28}=-\left(Y_{2}-Y\right), & j_{29}=-\left(Z_{2}-Z\right) \\
j_{10}=-\left(X_{3}-X\right), & j_{11}=-\left(Y_{3}-Y\right), & j_{12}=-\left(Z_{3}-Z\right) .
\end{array}\right.
$$

The values $\{X, Y, Z\}$ appearing in the Jacobi matrices $\boldsymbol{J}_{x}, \boldsymbol{J}_{y}$ are obtained from the closed form solution using either (3- 


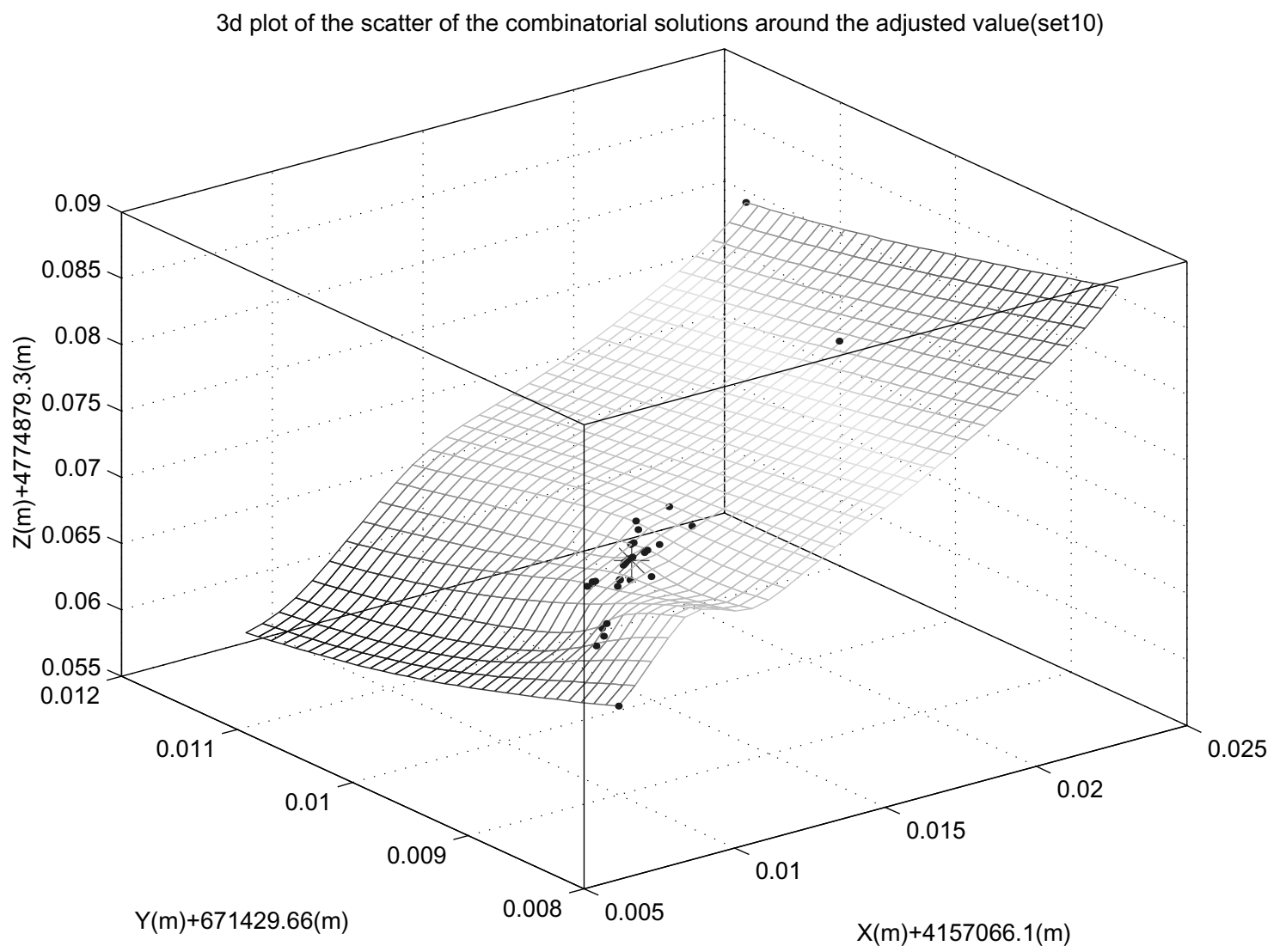

Fig. 4. Scatter of combinatorial solutions.

12) and (3-12) or (3-14) and (3-15). From the dispersion matrix $\boldsymbol{\Sigma}_{y}$ of the vector of observations $\boldsymbol{y}$ and with (3-17) and (3-18) forming $\boldsymbol{J}=\boldsymbol{J}_{x}^{-1} \boldsymbol{J}_{y}$, the variance-covariance matrix $\boldsymbol{\Sigma}_{x}$ is finally obtained from (3-2) as

$$
\begin{aligned}
& {\left[\begin{array}{ccc}
\sigma_{X}^{2} & \sigma_{X Y} & \sigma_{X Z} \\
\sigma_{Y X} & \sigma_{Y}^{2} & \sigma_{Y Z} \\
\sigma_{Z X} & \sigma_{Z Y} & \sigma_{Z}^{2}
\end{array}\right]} \\
& \left.=\boldsymbol{J} \begin{array}{cccccccccccc}
\sigma_{S_{1}}^{2} & \sigma_{S_{1} S_{2}} & \sigma_{S_{1} S_{3}} & 0 & 0 & 0 & 0 & 0 & 0 & 0 & 0 & 0 \\
\sigma_{S_{2} S_{1}} & \sigma_{S_{2}}^{2} & \sigma_{S_{2} S_{3}} & 0 & 0 & 0 & 0 & 0 & 0 & 0 & 0 & 0 \\
\sigma_{S_{3} S_{1}} & \sigma_{S_{3} S_{2}} & \sigma_{S_{3}}^{2} & 0 & 0 & 0 & 0 & 0 & 0 & 0 & 0 & 0 \\
0 & 0 & 0 & \sigma_{X_{1}}^{2} & 0 & 0 & 0 & 0 & 0 & 0 & 0 & 0 \\
0 & 0 & 0 & 0 & \sigma_{Y_{1}}^{2} & 0 & 0 & 00 & 0 & 0 & 0 & 0 \\
0 & 0 & 0 & 0 & 0 & \sigma_{Z_{1}}^{2} & 0 & 0 & 0 & 0 & 0 & 0 \\
0 & 0 & 0 & 0 & 0 & 0 & \sigma_{X_{2}}^{2} & 0 & 0 & 0 & 0 & 0 \\
0 & 0 & 0 & 0 & 0 & 0 & 0 & \sigma_{Y_{2}}^{2} & 0 & 0 & 0 & 0 \\
0 & 0 & 0 & 0 & 0 & 0 & 0 & 0 & \sigma_{Z_{2}}^{2} & 0 & 0 & 0 \\
0 & 0 & 0 & 0 & 0 & 0 & 0 & 0 & 0 & \sigma_{X_{3}}^{2} & 0 & 0 \\
0 & 0 & 0 & 0 & 0 & 0 & 0 & 0 & 0 & 0 & \sigma_{Y_{3}}^{2} & 0 \\
0 & 0 & 0 & 0 & 0 & 0 & 0 & 0 & 0 & 0 & 0 & \sigma_{Z_{3}}^{2}
\end{array}\right]
\end{aligned}
$$

with the $3 \times 3$ elements of $\boldsymbol{\Sigma}_{y}$ on the right hand side of (319) given from error propagation. The variance-covariance matrix computed as explained above is obtained for every combinatorial set $j=1, \ldots, 35$. Given $\boldsymbol{J}_{i}=\boldsymbol{J}_{x_{i}}^{-1} \boldsymbol{J}_{y_{i}}$ from the $i$-th combination and $\boldsymbol{J}_{j}=\boldsymbol{J}_{x_{j}}^{-1} \boldsymbol{J}_{y_{j}}$ from the $j$-th combination, the correlation between the $i$-th and $j$-th combination is given by

$$
\boldsymbol{\Sigma}_{i j}=\boldsymbol{J}_{j} \boldsymbol{\Sigma}_{y_{j} y_{i}} \boldsymbol{J}_{i}^{\prime}
$$

Finally we obtained the dispersion matrix $\boldsymbol{\Sigma}$ from the submatrices variance-covariance matrix for the individual combinatorials $\boldsymbol{\Sigma}_{1}, \boldsymbol{\Sigma}_{2}, \boldsymbol{\Sigma}_{3}, \ldots, \boldsymbol{\Sigma}_{k}$ (where $k$ is the number of combinations) obtained via (3-2) and the correlations between combinatorials obtained from (3-20) as

$$
\boldsymbol{\Sigma}=\left[\begin{array}{ccccc}
\boldsymbol{\Sigma}_{1} & \boldsymbol{\Sigma}_{12} & . & \ldots & \boldsymbol{\Sigma}_{1 k} \\
\boldsymbol{\Sigma}_{21} & \boldsymbol{\Sigma}_{2} & . & \ldots & \boldsymbol{\Sigma}_{2 k} \\
\cdot & & \boldsymbol{\Sigma}_{3} & \\
\cdot & & & \cdot & \\
\cdot & & & \cdot & \boldsymbol{\Sigma}_{k}
\end{array}\right]
$$

for the entire $k$ combinations.

Step 3: (Rigorous adjustment of the combinatorial solution points in a polyhedron):

For each of the 35 computed coordinates of point $\mathrm{K} 1$ in Fig. 3 in Step 2, we write the observation equations as

$$
\left[\begin{array}{c}
X^{j}=X+\varepsilon_{X}^{j} \mid, j \in\{1,2,3,4,5,6,7, \ldots, 35\} \\
Y^{j}=Y+\varepsilon_{Y}^{j} \mid j \in\{1,2,3,4,5,6,7, \ldots, 35\} \\
Z^{j}=Z+\varepsilon_{Z}^{j} \mid, j \in\{1,2,3,4,5,6,7, \ldots, 35\} .
\end{array}\right.
$$

With the values $\left\{X^{j}, Y^{j}, Z^{j}\right\}$ treated as pseudo-observation and placed in the vector of observation $y$, the coefficients of the unknown position $\{X, Y, Z\}$ being placed in the coefficient matrix $\boldsymbol{A}$ and $\boldsymbol{x}$ comprising the vector of unknowns 
Table 4. Position of station $K_{1}$ computed by Gauss-Jacobi combinatorial algorithm.

\begin{tabular}{ccccccc}
\hline Exp No. & $X(m)$ & $Y(m)$ & $Z(m)$ & $\sigma_{X}$ & $\sigma_{Y}$ & $\sigma_{Z}$ \\
\hline 1 & 4157066.1121 & 671429.6694 & 4774879.3697 & 0.00005 & 0.00001 & 0.00005 \\
\hline
\end{tabular}

Table 5. Deviation of the computed position of $K_{1}$ in Table 2 from the real measured GPS value.

\begin{tabular}{cccc}
\hline Exp No. & $\Delta X(m)$ & $\Delta Y(m)$ & $\Delta Z(m)$ \\
\hline 1 & -0.0005 & -0.0039 & 0.0007 \\
\hline
\end{tabular}

$\{X, Y, Z\}$, The solution is obtained via (3-3) and the dispersion of the estimated parameters through (3-4).

In the experiment above the computed position of point $K_{1}$ using the Gauss-Jacobi combinatorial approach is given in Table 4 with the deviation of the Gauss-Jacobi combinatorial solutions from the true (measured) GPS value given in Table 5. Figure 4 indicates the plot of the combinatorial scatter $\{\bullet\}$ around the adjusted values $\{*\}$.

\section{Conclusion}

For problems that require the solution of overdetermined ranging, and whose initial starting values are not known such as in photogrammetry, the Gauss-Jacobi combinatorial algorithm offers an alternative approach provided the full information of the underlying observation is taken into consideration via the nonlinear variance-covariance/error propagation. The advantage of the Gauss-Jacobi combinatorial being that no starting values, linearization or iterations is required as is the case with other procedures. Outlying combinations and observations are also identifiable. With high processing computers currently available, the issue of many combinatorials formed as a result of large observations is immaterial nor is the computing time. Routines are written that repeatedly execute the desired task once the statement that executes the combination has been written.

Acknowledgments. The first author wishes to acknowledge the support of JSPS (Japan Society of Promotion of Science) for the financial support that enabled the undertaking of this study. The author is further grateful for the support and the good working atmosphere provided by his hosts and co-authors professors S. Takemoto and Y. Fukuda of the Department of Geophysics, Graduate School of Science, Kyoto University, Japan.

\section{References}

Awange, J. L., Groebner basis solution of planar resection, Survey Review, 36(285), 528-543, 2002.
Awange, J. L., Diagnosis of Outlier of type multipath in GPS Pseudo-range observations, Survey Review, in press.

Awange, J. L., Algebraic approach to nonlinear global minimization problem relevant to Earth Sciences, Journal of Symbolic Computations, submitted.

Awange, J. L. and E. Grafarend, Algebraic solution of GPS pseudo-ranging equations, Journal of GPS Solutions, 4(5), 20-32, 2002a.

Awange, J. L. and E. Grafarend, Nonlinear adjustment of GPS observations of type pseudo-range, Journal of GPS Solutions, 4(5), 80-93, 2002b.

Awange, J. L. and E. Grafarend, Explicit solution of the overdetermined three-dimension resection problem, Journal of Geodesy, 76, 605-616, 2003.

Awange, J. L. and E. Grafarend, Polynomial optimization of the 7-parameter datum transformation problem when only three stations in both systems are given, Zeitschrift fuer Vermessungswesen, in press.

Awange, J. L., E. Grafarend, Y. Fukuda, and S. Takemoto, Direct polynomial approach to nonlinear distance (ranging) problems, Earth Planets Space, 55(5), 231-241, 2003.

Grafarend, E. and B. Schaffrin, The geometry of nonlinear adjustmentthe planar trisection problem-, in Festschrift to T. Krarup, edited by E. Kejlso, K. Poder, and C. C. Tscherning, pp. 149-172, Geodaetisk Institut, Meddelese No. 58, Denmark, 1989.

Grafarend, E. and B. Schaffrin, The planar trisection problem and the impact of curvature on non-linear least-squares estimation, Computational statistics \& data analysis, 12, 187-199, 1991.

Guolin, L., Nonlinear curvature measures of strength and nonlinear diagnosis, Allgemein Vermessungs-Nachrichten, 107, 109-111, 2000

Jurisch, R., G. G. Kampmann, and I. Schmadel, The Spatial Reference System (SRS) - an industrial application for geometric quality control introducing modern optimization, regulation and adjustment techniques, paper to be presented at the International Dimensional Workshop 2003, Nashville Marriott Nashville, Tennessee, U.S.A. May 12-16, 2003, (also available in pdf format at http: //www . metronomus. com/files/inora/idw_paper. PDF).

Kahmen, H. and W. Faig, Surveying, 578 pp, Walter de Gruyter, Berlin, 1988.

Krarup, T., Nonlinear adjustment and curvature, in Forty Years of Thought pp. 145-159, Delft, 1982.

Lohse, P., Ausgleichungsrechnung in nichtlinearen Modellen. DGK, Reihe C, Heft Nr. 429, 1994.

Mautz, R., Zur Lung nichtlinearer Ausgleichungsprobleme bei der Bestimmung von Frequenzen in Zeitreihen, DGK, Reihen C, Nr. 532, 2001.

Teunissen, P., Nonlinear least squares, Manuscripta Geodaetica, 15, 137 150, 1990.

$\mathrm{Xu}$, P., A hybrid global optimization method: the one-dimensional case, Journal of Computation and Applied mathematics, 147, 301-314, 2002.

$\mathrm{Xu}, \mathrm{P}$. , A hybrid global optimization method: the multi-dimensional case, Journal of Computation and Applied mathematics, 155, 423-446, 2003.

J. L. Awange (e-mail: awange@kugi.kyoto-u.ac.jp), Y. Fukuda, S. Takemoto, I. L. Ateya, and E. W. Grafarend (e-mail: grafarend@gis.unistuttgart.de) 